\title{
ON A SELF-TUNING MULTICAST FLOW CONTROL SCHEME-BASED AUTONOMIC TECHNOLOGY
}

\author{
N. Xiong ${ }^{1,2}$, Yanxiang $\mathrm{He}^{1}$, Yan Yang ${ }^{2}$, Laurence T. Yang ${ }^{3}$, Chao Peng ${ }^{2}$ \\ ${ }^{1}$ The State Key Lab of Software Engineering, Computer School of Wuhan University, PR.China. \\ ${ }^{2}$ School of Information Science, Japan Advanced Institute of Science and Technology, Japan. \\ ${ }^{3}$ Department of Computer Science, St. Francis Xavier University, Antigonish, B2G 2W5, Canada. \\ Email: naixue@jaist.ac.jp, yxhe@whu.edu.cn, lyang@stfx.ca, p.chao@jaist.ac.jp;
}

\begin{abstract}
With the increase of multicast data applications, research interests have focused on the design of congestion control schemes for multicast communications. This paper describes a novel control-theoretic multicast congestion control scheme, which is based on the distributed Self-tuning proportional plus integrative (SPI) controller. The control parameters can be designed to ensure the stability of the control loop in terms of source rate. The distributed explicit rate SPI overcomes the vulnerability that suffers from the heterogeneous multicast receivers. It is suggested that the congestion controller is located at the multipoint-to-multipoint multicast source to regulate the transmission rate. We further analyze the theoretical aspects of the proposed algorithm, and show how the control mechanism can be used to design a controller to support multicast transmissions. Simulation results demonstrate the efficiency of the proposed scheme in terms of system stability and fast response of the buffer occupancy, as well as controlled sending rates, low packet loss, and high scalability.
\end{abstract}

Index Terms - Buffer occupancy, explicit rate, multicast, rate-based congestion control, and stability.

\section{INTRODUCTION}

$\mathrm{W}$ ith the increase of multicast data applications, multicast (multipoint-to-multipoint) transmission now plays a key role in many applications such as teleconferencing and information dissemination services. Multicast improves the efficiency of multipoint data distribution by building a distribution tree from a sender to a set of receivers [1-2]. However, the widely used multicast transport protocols, which are layered on top of IP multicast, can cause congestion or even congestion collapse if they do not provide adequate congestion control. Congestion control thus plays an important role in the traffic management of multicast communications. Without an adequate congestion control scheme being implemented in a multicast tree, the incoming traffic to a bottleneck link might be much more than the outgoing link capacity, which could subsequently cause the buffer to overflow, and cause excessive queuing delay or even deadlock in certain nodes.

There are a great many congestion schemes handling unicast transmissions efficiently [11, 21, 22], and it was formulated as a discrete-time feedback control problem with delays. This control-theoretic approach to explicit rate control for ABR service was further analyzed and verified using a real network test-bed in the work by Kolarov and Ramamurthy [12]. Further achievements in this regard can also be found in [13-15]. All these methods are efficient in rate allocation and congestion control to unicast transmission. Unfortunately, multicast congestion control is much more sophisticated than that of unicast due to the complexity of multicasting mechanism.

Several multicast congestion approaches have been proposed recently. One class of them [3-5] adopts a simple hop-by-hop feedback mechanism, in which the feedback, i.e., backward control packets (BCPs), from downstream nodes are initially gathered at branch points, and then are transmitted upwards by a single hop upon receipt of a forward control packet (FCP). This kind of manipulation can be carried out on the basis of the tree structure in a multicast transmission. The main merit of these methods lies in the simplicity of the hop-by-hop mechanism; however at the same time, they often lead to the so-called consolidation noise problem [6-7] due to incomplete feedback information. To overcome this drawback, the papers [7-8] proposed a method called feedback synchronization at each branch point by accumulating feedback from all downstream branches. These schemes then introduce another problem of slow transient response due to the feedback from "long" path. Such delayed congestion feedback can cause excessive queue build-up and packet loss at the bottleneck link.

The authors of [9, 23] suggested that only a suitable set of representatives instead of all receivers send feedback to the sender, and the authors of [16] proposed a fuzzy-logic-based consolidation algorithm to estimate the unknown congestion information caused by long propagation delay. More recently, Zhang et al. [10] proposed an optimal second-order rate control algorithm to deal with control packet RTT (round-trip time) variations in multicast communications. This method has studied the system dynamics by using the binary congestion feedback in the scenarios of both persistent and on-off elastic traffic services, which defines that the data transfer rate is adjusted at the source depending on the available bandwidth at the bottleneck.

The major difficulty in designing of multicast congestion control protocols arises from the long and heterogeneous RTTs involved in the closed-loop control. Deep research is still called for in the following three aspects. First, the existing algorithms 
usually lack scalability since they require each router to keep maintaining the saturation status of every session (a session is composed of a multicast source, the corresponding transfer path and the corresponding switch node) and virtual session (VS) traveling through it, subsequently yielding a major computational bottleneck. To this end, we are going to present an algorithm that is scalable. Secondly, the known congestion control methods usually do not have explicit control over link buffer occupancy; as a consequence, the allocated rate can wander considerably before converging and link flow can temporarily exceed the capacity. To attack this problem, we will focus on the stability of our rate control scheme. Thirdly, no explicit rate allocation has been given in the known approaches. This paper will consider ER-based rate control with an explicit-rate feedback (ER-value). Such an ER-based scheme is responsive to network congestion and can serve WAN environments quite well where the bandwidth-delay product is usually large.

In this paper, we develop a distributed ER allocation algorithm to overcome the vulnerability that suffers from the heterogeneous multicast receivers. In our scheme, congestion controllers regulate the source rate at a multicast tree, which accounts for the buffer occupancies of all destination nodes. The proposed control scheme uses a distributed Self-tuning proportional plus integrative (SPI) controller, where the control parameters can be designed to ensure the stability of the control loop in terms of source rate. We further show how the control mechanism can be used to design a controller to support multipoint-to-multipoint multicast transmission based on feedback of ER's. System stability criterion is derived in presence of destination nodes with heterogeneous RTTs. We analyze the theoretical aspects of the proposed algorithm and verify its agreement with the simulations in the case of a bottleneck link appearing in a multicast tree. Simulation results demonstrate the efficiency of the proposed scheme in terms of system stability and fast response of the buffer occupancy, as well as controlled sending rates, low packet loss, and high scalability.

The rest of this paper is organized as follows. In section II, we present the congestion control network model. Section III describes the specific algorithm of SPI congestion control method. In section IV, we analyze the stability of the SPI controller. Section V presents various simulation results to validate and evaluate the performance of our scheme. Finally, in section VI, we present our conclusions and describe future work.

\section{THE NETWORK MODEL}

The multicast network is connection-oriented, composed of sources and destination nodes, and time is slotted with the duration $[n, n+1)$ by the sampling period $T$. The associated data is transferred by a fixed size packet.

In every sampling period, the multicast source issues and transmits forward control packets (FCPs) to the downstream nodes (the branch node and destination nodes), while backward control packets (BCPs) are constructed by the downstream nodes and sent back to the source.

After the multicast source receives the BCPs from the downstream nodes, it takes appropriate action to adjust its transmitting rates for multicast traffic based on the computed value of the SPI controller.

The branch node of the multicast session replicates each data packet, including the FCP, and transfers these packets to all its downstream nodes. Moreover, the branch nodes consolidate the BCPs that carry all the available rates and the relevant link bandwidth from different branches into one BCP and feedback the new BCP to their upstream node.

After receiving the data packets coming from the network, the receivers construct the BCPs and send them back to the network.

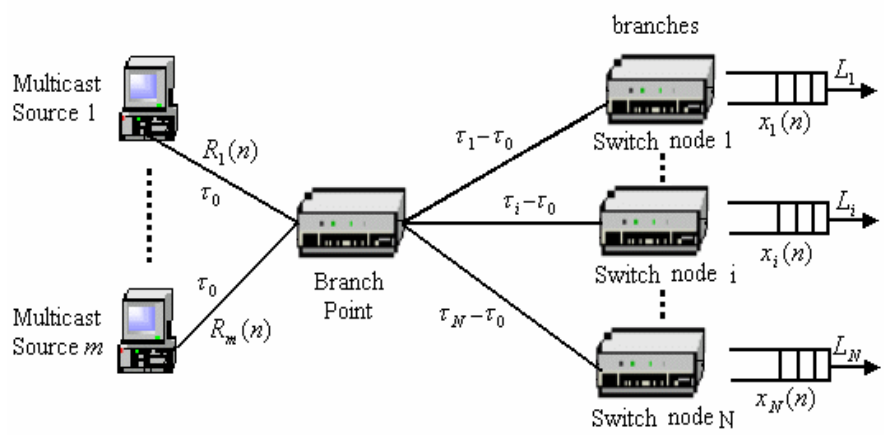

Figure 1. A multicast configuration of multiple points to multiple points

To conveniently analyze the performance and characteristics of the proposed multicast scheme, we focus on the following net work model [16] as shown in Figure 1, where the considered multicast service is described as follows:

(1) The packet number being sent out by switch node $i$ in one interval $T$ is denoted by $L_{i}$ [17], the switch node $i$ has the forward delay $\tau_{i}$, $(1 \leq i \leq N)$ from sources, and $\tau_{0}$ is the delay from sources to their neighbor downstream node. Then, the round-trip delay (RTD) for the switch node $i$ is $\tau_{R i}=2 \tau_{i}$, and $\tau=\max \left(\tau_{R 1}, \tau_{R 2}, \cdots, \tau_{R N}\right)$. We further assume that $\tau_{i}$ and $\tau_{R i}$ are integers, which are reasonable by adjusting $T$. The link delay is dominant compared to the other delays such as proceeding delay, queuing delay, etc.

(2) In the model, we assume $\tau_{i} \leq \tau_{j}$, when $1 \leq i \leq j \leq N$.

(3) Each router schedules the packets in a first-come-first-served way. The component $R_{i}(n)$ represents the receiving rate of the computed receivers $i$ at time slot $n$.

With the above notations and assumptions, the buffer occupancy of the switch node $i$ is determined by $[10,16]$ :

$$
x_{i}(n+1)=\operatorname{Sat}_{K_{i}}\left\{x_{i}(n)+\sum_{q=1}^{m} e_{q} R_{q}\left(n-\tau_{i}\right)-L_{i}\right\},
$$

where $K_{i}$ is the buffer size, $x_{i}(n)$ is the buffer occupancy of the switch node $i$ at time slot $n$, and $R_{q}\left(n-\tau_{i}\right)$ is the sending rate of the $q^{\text {th }}(1 \leq q \leq m)$ source to the switch node $i$, 


$$
(1 \leq i \leq N)
$$

$$
\begin{gathered}
\text { Sat } K_{i}\left\{x_{i}\right\}= \begin{cases}K_{i}, & x_{i}>K_{i} ; \\
x_{i}, & 0 \leq x_{i} \leq K_{i} ; \\
0, & x_{j i}<0 ;\end{cases} \\
e_{q}=\left\{\begin{array}{l}
1 ; \text { if the } q^{\text {th }} \text { source is active; } \\
0 ; \text { if the } q^{\text {th }} \text { source is not active; }
\end{array}\right.
\end{gathered}
$$

After lifting the saturation restriction [11], equation (1) can be written into

$$
x_{i}(n+1)=x_{i}(n)+\sum_{q=1}^{m} e_{q} R_{q}\left(n-\tau_{i}\right)-L_{i}
$$

\section{THE SPECIFIC ALGORITHM}

The key component of the proposed congestion control algorithm is the method for computing the required match between the source rate and the destinations' buffer. If $x_{i}(n)$ is too high, it can lead to buffer overflow and packet loss. If $x_{i}(n)$ is too low, it increases the likelihood of link under-utilization during occasionally idle periods. Thus the router buffer occupancy plays an important role in congestion control. In this paper, we first propose the following SPI controller, which is located at the sources and is updated upon every $T$ epoch.

$$
R_{q}(n)=\mu+a \cdot \sum_{i=1}^{N}\left(x_{i}\left(n-\tau_{i}\right)-\bar{x}_{i}\right)+\sum_{j=1}^{\tau} b_{j} R_{q}(n-j),
$$

where $a, b_{j}(j=1,2, \cdots, \tau)$ are the proportional and integral control gains respectively, which are to be determined from the stability criteria. These coefficients are used to locate all the poles of the closed-loop equations (2) and (3) within the unit circle to ensure stability. The component $\bar{x}_{i}$ is the target queue length, and $\mu$ is the maximum sending rate of sources. In (3) it is seen that, if the buffer occupancy of the switch node $i$ is measured at the instances $n-\tau_{i}$, after the feedback delay $\tau_{i}$ the BCP reaches the controller located at the source $q$, $(q=1,2, \cdots, m)$, and the router then takes out the buffer occupancy of the destination nodes at time $t=n$. In this way, the designed controller can be expected to have flexibility to cope with the sharp oscillation in buffer occupancy that could cause the network to lose packets. In addition, the calculation in (3) is completely independent of virtual connections traveling through the multicast session. This means the scheme has scalability.

Figure 1 depicts the proposed congestion control model for multiple points to multiple points in a multicast network. In the forward direction (from source to the destination nodes), a multicast flow is forwarded onto all of its outgoing links via the branch point. Rate adaptors associated are located at multicast source. There is a single FIFO (first-in-first-out) queue to multiplex all flows traveling through the outgoing link. The source of a multicast session issues and transmits a FCP in the forward direction repeatedly upon every transmission of data packets, in order to communicate flow-control related information with the routers in the session. The FCPs are also multicasted as data packets. The destination nodes of the multicast session send these control packets back to the source as soon as they receive them. These control packets in the backward direction are BCPs. The feedback consolidation algorithm runs at branch point in the backward direction. It merges the BCPs received from different branches into one BCP.

Before we present the algorithm in detail, we specify the following variables. The data multicasttree $[i]=1(0)$ means the $i^{\text {th }}$ branch points receive (don't receive) a FCP or BCP control packet ; while receivertree $[j]=1(0)$ means the $j^{\text {th }}$ branch points receive (don't receive) confirmations of all destination nodes. D means the number of data packets every $\mathrm{T}$ epoch. Based on the above specifications, the pseudo-code of the proposed router and source algorithms is given in Figure 2.

\section{SYSTEM STABILITY ANALYSIS}

We use a rate-based rather than a window-based adaptation algorithm to achieve congestion control in MR-MCC tree. The window-based scheme has extra complexity in maintaining and synchronizing the congestion window across all receivers, and it usually generates data bursts periodically [18]. In our proposed SPI control scheme, the rate adaptation takes into account the buffer occupancies of the destination nodes as well as the variation of RTTs. The controller parameters are designed to guarantee a stable of rate, which ensures a smooth dynamic of rate adaptation to minimize the packet loss rate. This is an obvious advantage of the proposed scheme over the widely adopted AIMD (addictive increase and multiplicative decrease) method (see, for example [18]). For example, in AIMD, it is difficult to choose the appropriate increase and decrease factors to guarantee the system's stability and then to obtain smooth and healthy rate adaptation and good link utilization.

In this section, the stability of the proposed SPI congestion control scheme is analyzed as follows. Considering the equation (2), if z-transformation is applied, one can easily arrive at

$$
(z-1) X_{i}(z)=\sum_{q=1}^{m} e_{q} R_{q}(z) z^{-\tau_{i}}-L_{i} D(z),
$$

where the z-transformation of $x_{j i}(n), R_{j}(n)$ are respectively described by $X_{i}(z)=\sum_{n=0}^{+\infty} x_{i}(n) z^{-n}, R_{q}(z)=\sum_{n=0}^{+\infty} R_{q}(n) z^{-n}$, and $D(z)=\sum_{n=0}^{+\infty} z^{-n}=\frac{z}{z-1}$.

Taking the z-transform of equation (3), one yields:

$$
\begin{aligned}
R_{q}(z)= & \mu \cdot D(z)+a \cdot \sum_{i=1}^{N}\left(z^{-\tau_{i}} \cdot X_{i}(z)-\bar{x}_{i} \cdot D(z)\right) \\
& +\sum_{j=1}^{\tau} b_{j} \cdot z^{-j} \cdot R_{q}(z)
\end{aligned}
$$

From equation (4) and (5), one has 


$$
\begin{aligned}
\Delta z R_{q}(z) & =a \sum_{i=1}^{N}\left(-L_{i} D(z) z^{-\tau_{R i} / 2}-\bar{x}_{i} D(z)(z-1)\right) \\
& +\mu D(z)(z-1)
\end{aligned}
$$

\section{Source Algorithm}

Upon every $\mathrm{T}$ epoch (say, time $\mathrm{k}$ )

Transmit data including $F C P$;

Upon receipt of a consolidation BCP from it's downstream

Compute the sending rate based on consolidation $B C P$ using SPI controller;

Adjust the transmitting rates based on computed sending rate.

\section{Router Algorithm}

If multicasttree $[i]==1$

\{

if the packet is an FCP

\{

Put the data packet in the buffer;

Copy the data including FCP;

Multicast them to the downstream nodes;

\}

else

\{

Construct the BCP based on the received $B C P$;

Feedback it to the upstream node;

if receivedtree $[j]==1$

\{ Delete the data packets from the buffer; \}

else \{

Maintain the data packets in the buffer until receive all confirmations of the receivers; \}

\}

\}

\section{Destination Node Algorithm}

Upon receipt of an FCP

Put the data packets into the buffer;

Construct the BCP based on the current congestion condition of the receiver nodes;

Feedback the BCP to the upstream branch point.

Figure 2. Pseudo-code of source/ router/ destination node algorithms.

where we have denoted,

$$
\Delta z=\left(1-\sum_{j=1}^{\tau} b_{j} z^{-j}\right)(z-1)-a \sum_{i=1}^{N} z^{-\tau_{R i}}
$$

The coefficients $a$ and $b_{j},(j=1,2, \cdots, \tau)$ are determined by the stability criteria of the control theory.

The component $\Delta z$ is the Characteristic Polynomial (CP) of the multicast system given by equations (2) and (3) [19]. The
$\mathrm{CP}$ (6) is closely related to the stability of the congestion-controlled network system. From a control-theoretic view when all the zeros of (6) lie within the unit disc, the original network system (2) with the controller (3) is stable in terms of the source's sending rate. Stability is a prerequisite in congestion control to ensure that the network has no sending rate oscillation and thus minimize the packet loss rate.

The following computation procedures aid us in determining if all the roots of a characteristic polynomial lie within a unit disc. Without loss of generality, we group those nodes into one class, which has a small variation of time delays and sending rates. Thus we divide $N$ destination nodes into $M$ groups based on the RTTs, and in each group, the RTT is assumed to be equal, i.e.,

$$
\left\{\tau_{R 1}, \tau_{R 2}, \tau_{R 3}, \cdots, \tau_{R N}\right\}=\left\{t_{1}, \cdots, t_{1}, t_{2}, \cdots, t_{2}, \cdots, t_{M}, \cdots, t_{M}\right\}
$$

and we set $n_{i}$ as the number of the RTT $t_{i}(i=1,2, \cdots, M)$ corresponding to the $i^{\text {th }}$ group receivers, then $N=\sum_{i=1}^{M} n_{i}$ ).

And $n_{i}$ is positive integer. So the $\mathrm{CP}$ is:

$$
\begin{aligned}
& \Delta z=-z^{-\tau} \cdot\left[-z^{\tau+1}+\left(b_{1}+1\right) \cdot z^{\tau}+\left(b_{2}-b_{1}\right) \cdot z^{\tau-1}+\left(b_{3}-b_{2}\right) \cdot z^{\tau-2}+\right. \\
& \cdots+\left(b_{\tau_{R 1}}-b_{\left(\tau_{R 1}\right)-1}\right) z^{\tau-\left(\tau_{R 1}\right)+1}+\left(b_{\left(\tau_{R 1}\right)+1}-b_{\tau_{R 1}}-a \cdot n_{1}\right) \cdot z^{\left(\tau-\tau_{R 1}\right)}+ \\
& \left(b_{\left(\tau_{R 1}\right)+2}-b_{\left(\tau_{R 1}\right)+1}\right) \cdot z^{\tau-\left(\tau_{R_{1}}\right)-1}+\cdots+\left(b_{\tau_{R_{i}}}-b_{\left(\tau_{R_{i}}\right)-1}\right) \cdot z^{\tau-\left(\tau_{R_{i}}\right)+1}+\left(b_{\tau_{R_{i}}}\right. \\
& \left.-b_{\left(\tau_{R_{i}}\right)-1}-a \cdot n_{i}\right) \cdot z^{\tau-\left(\tau_{R_{i}}\right)}+\cdots+\left(b_{\tau_{R_{(M-1)}}}-b_{\left(\tau_{R_{(M-1)}}\right)-1}-a \cdot n_{(M-1)}\right) \\
& \left.\cdot z^{\tau-\left(\tau_{R(M-1)}\right)}+\cdots+\left(b_{\tau}-b_{\tau-1}\right) \cdot z+\left(b_{\tau}-a \cdot n_{M}\right)\right] .
\end{aligned}
$$

Let

$1+b_{1}=b_{2}-b_{1}=b_{3}-b_{2}=\cdots=b_{\tau_{R 1}}-b_{\left(\tau_{R 1}\right)-1}=b_{\left(\tau_{R 1}\right)+1}-b_{\tau_{R 1}}-a \cdot n_{1}$ $=b_{\left(\tau_{R 1}\right)+2}-b_{\left(\tau_{R 1}\right)+1}=\cdots=b_{\tau_{R_{i}}}-b_{\left(\tau_{R_{i}}\right)-1}=b_{\tau_{R_{i}}}-b_{\left(\tau_{R_{i}}\right)-1}-$ $a \cdot n_{i}=\cdots=b_{\tau_{R_{(M-1)}}}-b_{\left(\tau_{\left.\tau_{(M-1)}\right)}-1\right.}-a \cdot n_{(M-1)}=\cdots=b_{\tau}-b_{\tau-1}$, and $b_{\tau}=a n_{M}$. We set $a=(\tau \varepsilon-1) / N$, and $b_{j}=\left\{\begin{array}{lc}j \varepsilon-1 ; & \left(j \in\left[1, \tau_{R 1}\right]\right) ; \\ j \varepsilon-1-a \sum_{k=1}^{i} n_{k} ;(i \in[1, & \left.(M-1)], j \in\left[\tau_{\left(R_{i}\right)+1}, \tau_{R_{(i+1)}}\right]\right) .\end{array}\right.$ we can get

$$
\Delta(z)=-z^{-\tau}\left[-z^{\tau+1}+\varepsilon\left(z^{\tau}+z^{(\tau-1)}+z^{(\tau-2)}+\cdots+z\right)\right]
$$

From [20], when $\varepsilon<1 /(\tau+1)$, all the zeros of (7) lie within the unit disc, and the original network system (2) with the controller (3) is stable.

\section{THE SIMULATION RESULTS}

To evaluate the performance of the studied multicast congestion control method, we focus upon the following two simulation models and are mostly interested in analyzing the transient behaviors of the network. In the performance analysis, the duration of response time, receiving rate of receivers and steady state of buffer occupancy are the main concerns.

A control method with short response time has the following advantages: when the buffer of receiver nodes is close to the 
threshold, one may notify the sending node to reduce the sending rate and prevent the loss of packets as soon as possible; while when the available bandwidth increases, the sending node increases the sending rate as soon as possible and enhances the utilization rate of the bandwidth.

In simulations, we process the nodes, which have a small change of time delay and sending rate together. Then we unify the time delay and sending rate.

Since the situation of every node in each group (about 20 receivers) is similar, we only choose one node from each group as a representative. We assume that the link delay is dominant compared to the other delays such as processing delays and queuing delay.

Simulation is carried out over a wide range of patterns and propagation between two different nodes can lie in the LAN (Local Area Network) case or the WAN (Wide Area Network) case. Based on the complex degree of the mode and network dynamic behavior, we present two simulation experiments. In simulation 1 (see Figure 3), the multicast source S1 sends data packets at $0 \mathrm{~m} \mathrm{sec}$, and the multicast source S2 starts to sends data packets at $1000 \mathrm{~m} \mathrm{sec}$ in the simulation time; then it enhances the network dynamic behavior, and also demonstrates the efficiency of the SPI scheme. In simulation 2 (see Figure 4), we set $\varepsilon$ at two different values: one enables system stability, and the other is unstable in the same system. Thus, we compare and analyze the two cases in the same system.

\section{A. Simulation 1}

In this simulation, we focus on the simulation model (see Figure 3) and we are mostly interested in analyzing the transient behaviors of the network.

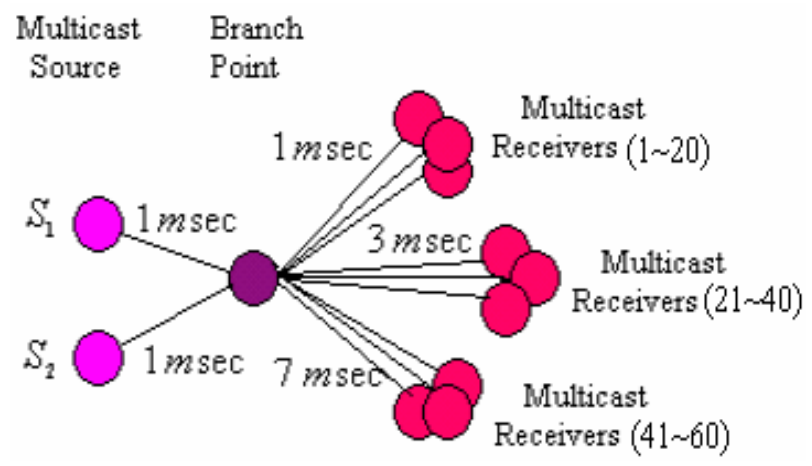

Figure 3. The multicast system simulation model 1.

The relevant notations and assumptions are listed in Table I. According to the introduced stability test to select the control gains, one computes the relevant parameters $a, b, c$.

For this case, $\tau=16 \mathrm{msec}, N=60, n_{1}=n_{2}=n_{3}=20$, we set $\varepsilon$ to be $1 / 20$, which is stable in the system. When $\varepsilon=1 / 20$,

$$
\begin{aligned}
a= & -1 / 300, \text { and } \\
b= & {\left[b_{1}, b_{2}, b_{3}, \ldots, b_{16}\right] } \\
= & {[-19 / 20,-9 / 10,-17 / 20,-8 / 10,-41 / 60,-38 / 60,} \\
& -35 / 60,-32 / 60,-25 / 60,-22 / 60,-19 / 60,-8 / 30, \\
& -13 / 60,-10 / 60,-7 / 60,-4 / 60] .
\end{aligned}
$$

Table I. The parameters in the simulation model 1

\begin{tabular}{|c|c|c|c|}
\hline $\begin{array}{c}\text { Receivers } \\
\text { Variables }\end{array}$ & Receiver 1 & Receiver 21 & Receiver 41 \\
\hline $\bar{x}_{i}(K b)$ & 70 & 80 & 120 \\
\hline$L_{i}(M b p s)$ & 2 & 3 & 4 \\
\hline$\tau_{R j i}(m \mathrm{sec})$ & 4 & 8 & 16 \\
\hline
\end{tabular}

The results of simulation 1 are shown in Figures 5 -9. Figures 7-9 show the buffer transient response of receiver node 1 , node 21, and node 41 respectively. These buffer occupancies all have some fluctuation in the beginning, then are gradually stabilized. The sending rates in the sources are respectively shown in Figures 5-6. The initial sending rate of multicast source $\mathrm{S} 1$ is $6 \mathrm{Mbps}$; As time goes on, the sending rate of source S1 is gradually adjusted and has some fluctuation, then the sending rate is quickly stable at the value of $2 \mathrm{Mbps}$ during $74 \mathrm{~m}$ sec. As the multicast source S2 starts to send data packets at $1000 \mathrm{~m} \mathrm{sec}$, then the sending rate of source S1 has some fluctuation, and quickly stabilizes at the new value of $1 \mathrm{Mbps}$. These simulation results demonstrate the efficiency of our scheme, in which the source adjusts the sending rate gradually to make the buffer occupancy and rate of sending node quickly become steady.

\section{B. Simulation 2}

In this simulation, we focus on simulation model 2 (see Figure 4) and compare the different parameters for stable and unstable systems.

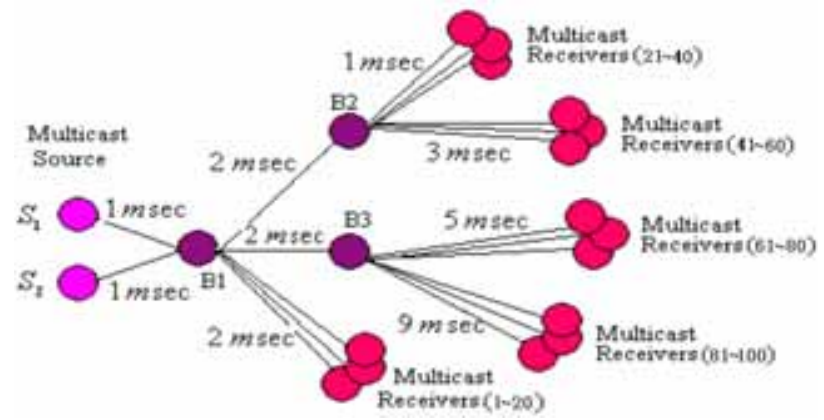

Figure 4. The multicast system simulation model 2.

The relevant network parameters and assumptions are listed in Table II. According to the introduced stability test to select the control gains, one computes the relevant parameters $a, b, c$.

For this case, $\tau=24 m \mathrm{sec}, N=100 \quad n_{1}=\cdots=n_{5}=20$, we separately set $\varepsilon$ to be $1 / 40$ and 0.9 . The first value is stable in the system, and the next one, $\varepsilon=0.9$, enables system to be unstable. When $\varepsilon=1 / 40, a=-1 / 250$, and

$$
\begin{aligned}
& b=\left[b_{1}, b_{2} \ldots, b_{24}\right] \\
& =[-39 / 40,-38 / 40,-37 / 40,-36 / 40,-35 / 40,-34 / 40, \\
& -149 / 200,-72 / 100,-123 / 200,-59 / 100,-113 / 200,-53 / 100, \\
& -87 / 200,-82 / 200,-77 / 200,-72 / 200,-51 / 200,-46 / 200, \\
& -41 / 200,-36 / 200,-31 / 200,-26 / 200,-21 / 200,-16 / 200] ;
\end{aligned}
$$

When $\varepsilon=0.9, a=0.206$, and 
$b=\left[b_{1}, b_{2} \ldots, b_{24}\right]$

$=[-0.1,0.8,1.7,2.6,3.5,4.4,5.38,6.28,7.26,8.16$,

9.06, 9.96, 10.94, 11.84, 12.74, 13.64, 14.62, 15.52,

$16.42,17.32,18.22,19.12,20.02,20.92]$.

Table II. The parameters in simulation model two

\begin{tabular}{|c|c|c|c|}
\hline $\begin{array}{c}\text { Receivers } \\
\text { Variables }\end{array}$ & $\tau_{\text {Rji }}(m \mathrm{sec})$ & $L_{i}(M b p s)$ & $\bar{x}_{i}(K b)$ \\
\hline Receiver 1 & 6 & 2 & 70 \\
\hline Receiver 21 & 8 & 2 & 75 \\
\hline Receiver 41 & 12 & 3 & 80 \\
\hline Receiver 61 & 16 & 4 & 120 \\
\hline Receiver 81 & 24 & 5 & 140 \\
\hline
\end{tabular}

As the two sources send data packets at same time, we only choose source S1 from each group as a representative. When the control gains enable system stability, we can see the short steady response time of the sending source and buffer occupancy conflicting with the high sending rate of the multicast source between the sending node and the branch nodes. The sending rate of multicast source S1 is shown in Figure 10. After $222 \mathrm{~m} \mathrm{sec}$, the sending rate in the stable system is stable at the value $1 \mathrm{Mbps}$. Figures 11-15 show buffer occupancies in Receivers 1, 21, 41, 61 and 81. In the stable case, the buffer occupancies in Receivers 1 and 21 are quickly stabilized at the values of $82.5 \mathrm{~Kb}$ and $86.5 \mathrm{~Kb}$ respectively (Figures 11-12). In Figures 13-15, some packets accumulate in the receivers' buffer in the beginning. As time goes on, the controller starts to adjust the transmission rate of the source, and the remaining packets in buffer are cleared. In the unstable system, the controller fails to effectively adjust the sending rate of sources to make the buffer occupancy stable.

\section{Simulation Results}

Based on simulations 1 and 2, we can obtain the simulation data in Table III.

The simulation results verify the theoretical agreement with the simulations in the case of a bottleneck link appearing in a multicast tree. The results demonstrate the efficiency of our scheme in terms of system stability, scalability, high utilization of bottleneck links, and fast response. The simulation results also demonstrate that the sources efficiently and quickly adjust the sending rate to make both the buffer occupancy of receivers and the sending rate of multicast sources become excellently steady. Therefore, this SPI scheme is an effective and efficient.

\section{CONCLUSIONS AND FUTURE WORK}

This paper presented the theoretical analysis and design of a multicast scheme, using an explicit rate feedback mechanism to design a SPI controller for regulating the source rates in multicast communication networks. The control parameters of the SPI controller can be designed to ensure the stability of the control loop in terms of buffer occupancy on the basis of control theory; this subsequently means that it accomplishes the least packet loss in steady state. Relevant pseudo-codes for implementation have been developed, and the paper shows how the SPI controller could be designed to adjust the rates of the data service. Simulations have been carried out with multipoint-to-multipoint multicast models. The simulation results clearly demonstrate the efficiency of our scheme in terms of system stability and fast response of the buffer occupancy, as well as controlled sending rates, low packet loss, and high scalability. As evident from the analyses and simulation results, the proposed multicast scheme is simple and also supports unicast. We believe that our work is a valuable foundation for a unified flow control scheme capable of being deployed in the real multicast networks.

Table III. The simulation results of the SPI controller

\begin{tabular}{|c|c|c|c|c|c|}
\hline \multicolumn{3}{|c|}{ Variables node } & \multirow{2}{*}{$\begin{array}{c}\text { S1 } \\
2 \\
M b p s\end{array}$} & \multirow{2}{*}{$\begin{array}{c}\mathrm{S} 2 \\
0\end{array}$} & \multirow{2}{*}{\begin{tabular}{|c|c|}
$\begin{array}{c}\text { Buffer } \\
\text { occupancy } \\
\text { of Receiver } 1\end{array}$ \\
$93 \mathrm{~Kb}$
\end{tabular}} \\
\hline 莺 & \multirow{2}{*}{ 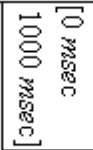 } & Steady value & & & \\
\hline 愛. & & $\begin{array}{c}\text { Response } \\
\text { time (msec) }\end{array}$ & 397 & 0 & 451 \\
\hline $\begin{array}{l}\stackrel{3}{\circ} \\
\stackrel{8}{\circledR 0}\end{array}$ & \multirow{2}{*}{ 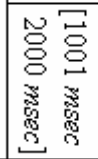 } & Steady value & $\begin{array}{c}1 \\
M b p s\end{array}$ & $\begin{array}{c}1 \\
M b p s\end{array}$ & $227 \mathrm{~Kb}$ \\
\hline$\underset{8}{\stackrel{8}{8}}$ & & $\begin{array}{c}\text { Response } \\
\text { time (msec) }\end{array}$ & 294 & 309 & 345 \\
\hline \multirow{2}{*}{ 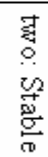 } & \multirow{2}{*}{ 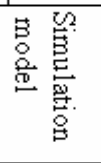 } & Steady value & $\begin{array}{c}1 \\
M b p s\end{array}$ & $\begin{array}{c}1 \\
M b p s\end{array}$ & $114 \mathrm{~Kb}$ \\
\hline & & $\begin{array}{c}\text { Response } \\
\text { time (msec) }\end{array}$ & 304 & 304 & 324 \\
\hline
\end{tabular}

Interesting future work will address issues related to TCP-friendliness in multicast flow control, following the same line of study.

\section{ACKNOWLEDGMENTS}

This research has been supported by the Japanese Government Monbukagakusho Foundation, by the COE (Strategic Development of Science and Technology) foundation in Japan, by the National Natural Science Foundation of China under Grant No. 90104005, by the Key Project of Natural Science Foundation of Hubei Province under Grant No. 2003ABA047, and by the foundation of the State Key Lab of Software Engineering.

We would like to thank the many colleagues and anonymous reviewers for who gave us constructive criticism and helpful suggestions for improving the overall quality of this paper.

\section{REFERENCES}

[1] S. Deering, Host extensions for IP multicasting. RFC1112, Jan. 1989.

[2] Rung-Hung Gau, Zygmunt J. Haas, Bhaskar Krishnamachari, "On Multicast Flow Control for Heterogeneous Receivers," IEEE/ACM Transactions on Networking, Volume 10, Issue 1, pp. 86-101, February 2002.

[3] L. Roberts, "Rate based algorithm for point to multipoint ABR service," ATM Forum, Contribution 94-0772, Sept. 1994.

[4] K. Y. Siu and H. Y. Tzeng, "On max-min fair congestion control for multicast ABR services in ATM," IEEE Journal on Selected Areas in Communications, vol. 15, pp. 545-556, April, 1997.

[5] H. Saito, K. Kawashima, H. Kitazume, A. Koike, M. Ishizuka, and A. Abe, "Performance issues in public ABR service," IEEE Communication 
Magazine, pp. 40-48, Nov. 1996.

[6] X. Zhang and K. G. Shin, "Statistical analysis of feedback synchronization signaling delay for multicast flow control," in Proc. IEEE INFOCOM, pp. 1152-1161, April 2001

[7] Y. Z. Cho, S. M. Lee, and M. Y. Lee, "An efficient rate-based algorithm for point-to-multipoint ABR service,” in Proc. IEEE GLOBECOM, pp. 790-795, Nov. 1997.

[8] W. Ren, K. Y. Siu, and H. Suzuki, "On the performance of congestion control algorithms for multicast ABR service in ATM," in Proc. IEEE ATM Workshop, Aug. 1996.

[9] D. DeLucia and K. Obraczka, "Multicast feedback suppression using representatives," in Proceedings of IEEE INFOCOM’97, 1997.

[10] Xi Zhang, Kang G. Shin, Debanjan Saha and Dilip D. Kandlur, "Scalable Flow Control for Multicast ABR Services in ATM Networks," IEEE/ACM Transactions on Networking, vol.10, no.1, pp 67-85, 2002.

[11] L. Benmohamed and S.M. Meekov, "Feedback control of congestion in packet switching networks: The case of a single congested node", IEEE/ACM Transactions on Networking, vol.1, pp. 693-708, December 1993.

[12] A. Kolarov and G. Ramamurthy, "A control theoretic approach to the design of an explicit rate controller for ABR service," IEEE/ACM Transactions on Networking, vol.7, pp. 741-753, October 1999.

[13] Liansheng Tan, A. C. Pugh and Min Yin. "Rate-Based Congestion Control in ATM Switching Networks Using a Recursive Digital Filter”, Control Engineering Practice (Special Issue on Control Methods for Telecommunication Networks), vol. 11, no. 10, pp. 1171-1181, 2003.

[14] F. Blanchini, R. Lo Cigno and R. Tempo, "Robust rate control for integrated services packet networks", IEEE/ACM Transactions on Networking, vol.10, no. 5, pp. 644-652, October, 2002.

[15] R. Jain, S. Kalyanaraman, R. Goyal, S. Fahmy, R. Viswanathan, "ERICA Switch Algorithm: A Complete Description”, ATM Forum-Tm 96-1172, Auguest 1996

[16] S. H. Lee and J. T. Lim, "Multicast ABR service in ATM networks using a fuzzy-logic-based consolidation algorithm”, IEE Proceeding Communication, Vol.148, No. 1, pp. 8-13, February 2001.

[17] The ATM forum Technical Committee Traffic Management Specification. Version 4.0, af-tm-0056.000, April 1996.

[18] S. Shi and M. Waldvogel, "A rate-based end-to-end multicast congestion control protocol," in Proceedings of Fifth IEEE Symposium on Computersand Communications (ISCC 2000), 2000.

[19] Naixue Xiong, Yanxiang He, Yan Yang, "An Efficient Flow Control Algorithm for Multi-rate Multicast Networks,” 2004 IEEE International Workshop on IP Operations \& Management (IPOM' 04), Beijing, China, pp. 69-76, October 11- 3, 2004.

[20] Liansheng Tan, A. C. Pugh and Min Yin, "Rate-Based Congestion Control in ATM Switching Networks Using a Recursive Digital Filter," Control Engineering Practice (Special Issue on Control Methods for Telecommunication Networks), Vol.11, No. 10, PP. 1171-1181, 2003.

[21]S.Keshav, “A control-theoretic approach to flow control,” In: Proceedings of ACM SIGCOMM’91, Zurich: ACM Press, pp. 3-15, 1991.

[22]Lotfi Benmohamed, Semyon M. Meerkov, "Feedback control of congestion in packet-switching networks: The case of multiple congested nodes," International Journal of Communication Systems, 10(5), pp. 227-246, 1997.

[23]L. Rizzo, "pgmcc: a TCP-friendly single-rate multicast congestion control scheme,” In: Proceedings ACM SIGCOMM 2000. Stockholm, pp.17-28, 2000.

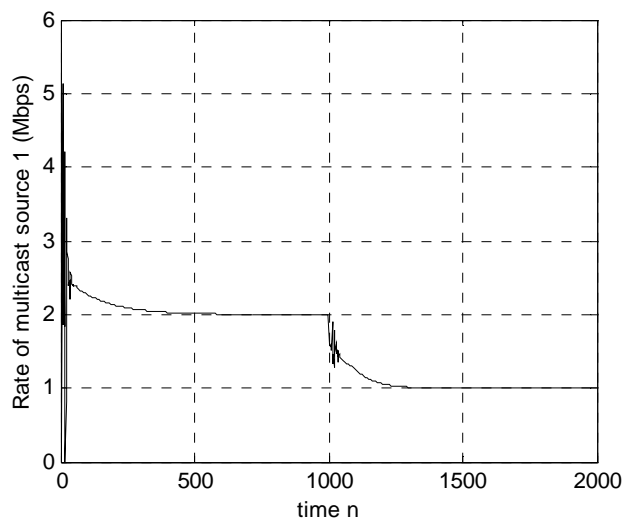

Figure 5. The sending rate of multicast source S1 (simulation model 1)

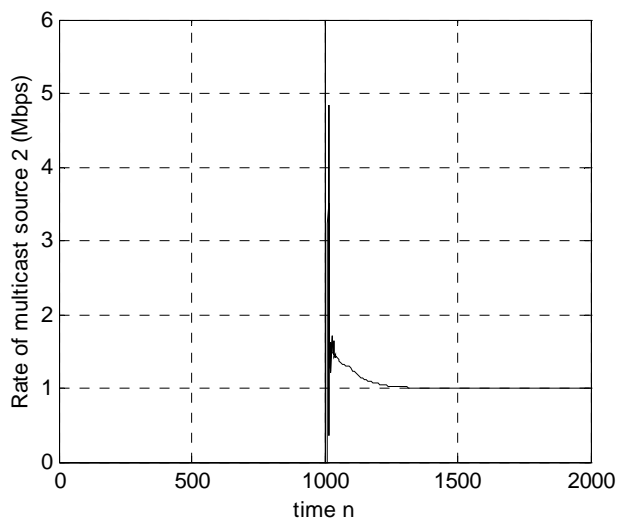

Figure 6. The sending rate of multicast source S2 (simulation model 1)

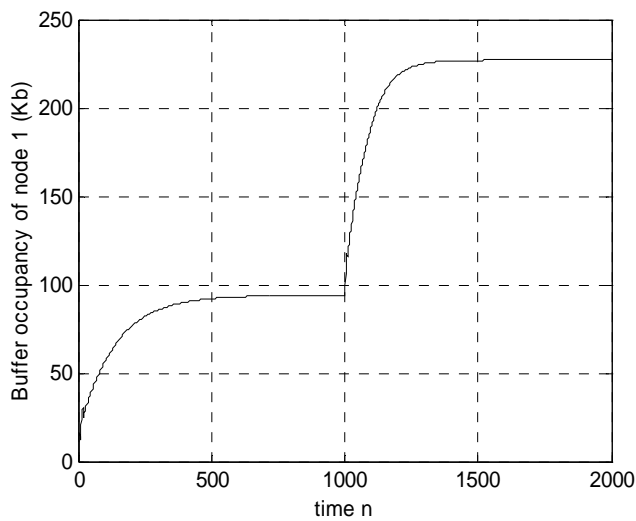

Figure 7. The transient response of buffer occupancy in receiver node 1 (simulation model 1)

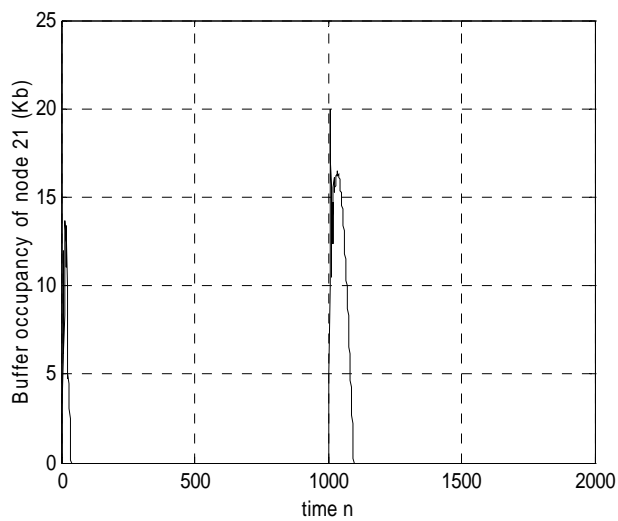

Figure 8. The transient response of buffer occupancy in receiver node 21 (simulation model 1) 


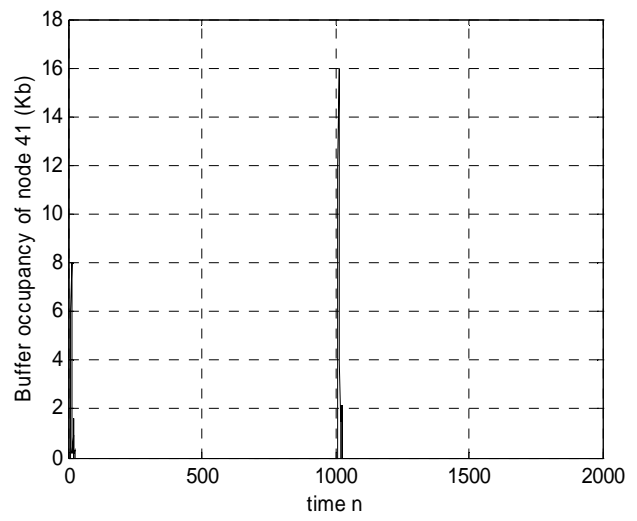

Figure 9. The transient response of buffer occupancy in receiver node 41 (simulation model 1)

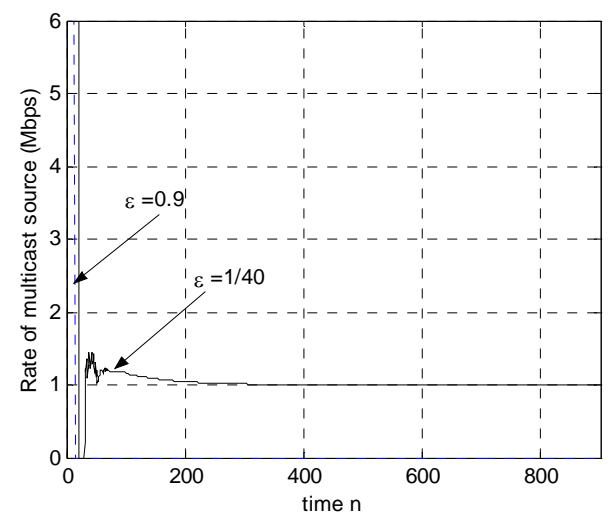

Figure 10. The sending rate of multicast source S1 (simulation model 2)

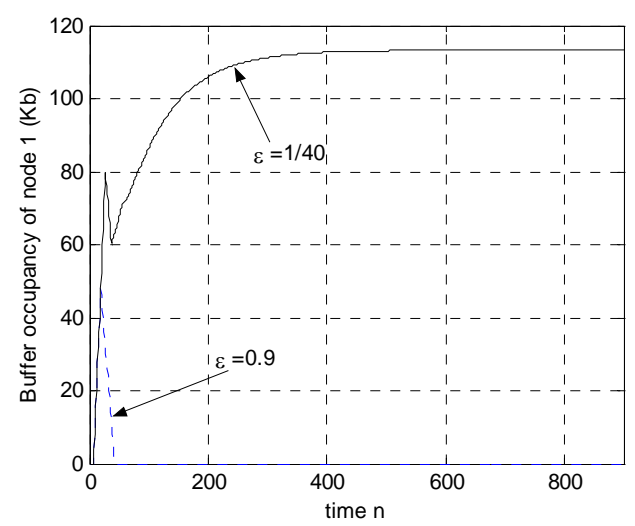

Figure 11. The transient response of buffer occupancy in receiver node 1 (simulation model 2)

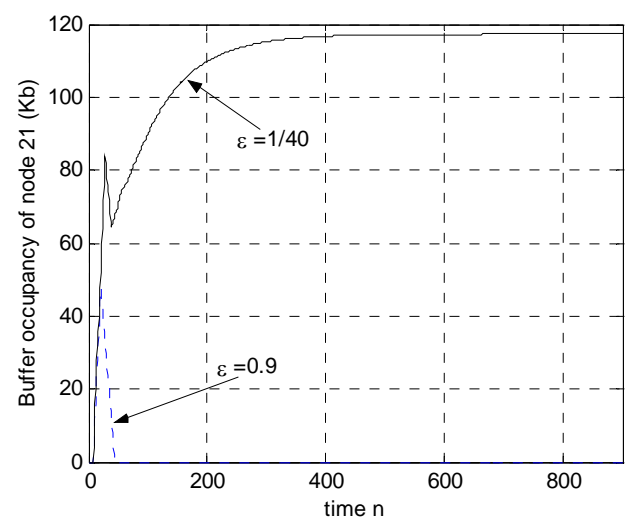

Figure 12. The transient response of buffer occupancy in receiver node 21 (simulation model 2)

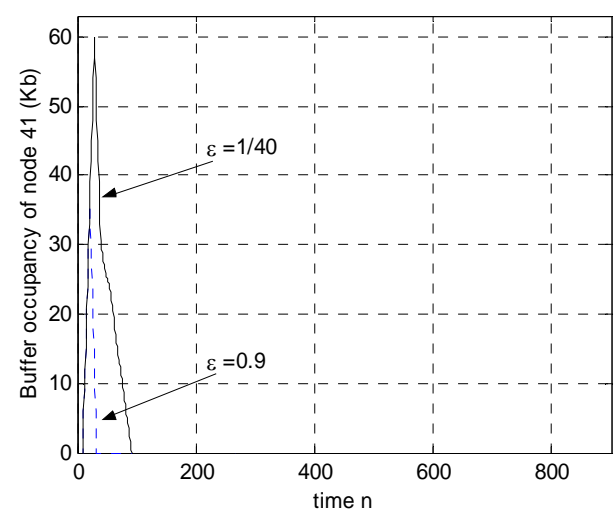

Figure 13. The transient response of buffer occupancy in receiver node 41 (simulation model 2)

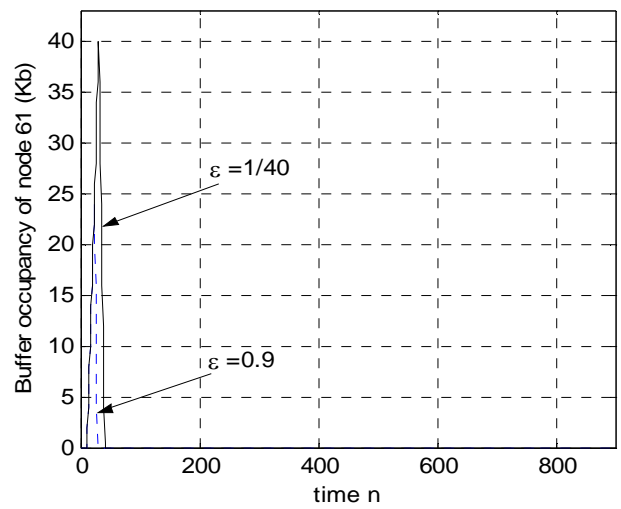

Figure 14. The transient response of buffer occupancy in receiver node 61 (simulation model 2)

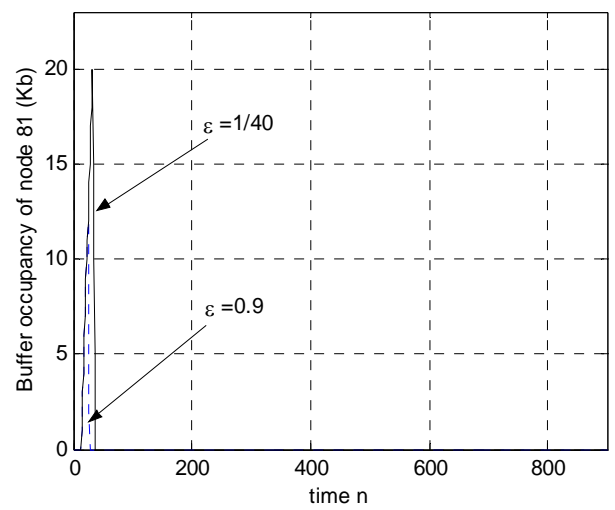

Figure 15. The transient response of buffer occupancy in receiver node 81 (simulation model 2) 